\title{
Caracterización estructural y funcional de una metaloproteasa del veneno de Bothrops roedingeri por aproximación proteómica
}

Structural and functional characterization of a metaloprotease of the venom of Bothrops roedingeri by proteomic approach

Juan Carlos Campos Gonzales, Luis Ángel Arenas Menéndez, Jaime Barreda del Carpio, Iván Paz Aliaga Universidad Católica de Santa María. Arequipa Perú.

\section{INFORMACIÓN}

\section{Historia del Artículo}

Recepción: 06/06/2018

Revisión: 20/11/2018

Aceptación: 18/01/2019

\section{Palabras Clave}

HITrap, Cromatografia, SDS PAGE, Espectrometría de Masas, ESI, MALDI/TOF, Hidrolisis, Actividad hemorrágica, Proteómica.

\section{Key Words}

HITrap, Chromatography, SDS PAGE, Mass Spectrometry, ESI, MALDI / TOF, Hydrolysis, Hemorrhagic activity, Proteomics.

\section{DOI}

https://doi.org/10.35286/veritas. v20i2.245

\section{RESUMEN}

Las especies pertenecientes al género Bothrops son muy importantes en el campo de la investigación por poseer enzimas proteolíticas, la cual tiene como características principales tener una variada acción biológica y contribuir con desórdenes en coagulación, necrosis y formación de hemorragias, por ende se necesita una plataforma moderna en el estudio de estas enzimas para así poder sugerir un modelo metodológico actualizado en el estudio de todas las proteínas expresadas por un sistema biológico que nos permita conocer el estado actual de la especie, así como utilizar esta información en la construcción biotecnológica de informaciones con posibles fines terapéuticos futuros. El primer objetivo trazado es purificar y caracterizar una de las enzimas proteolíticas presentes en el veneno como es la metaloproteasa para lo cual se trabajó inicialmente mediante cromatografía de Interacción Hidrofóbica con columnas del sistema HITrap en la cual nos dio como resultado la presencia de 6 picos, siendo el pico 2 aquel que mostró actividad hemorrágica siendo denominada $\mathrm{HFBr}$, con esta fracción obtenida se realizó una electroforesis SDS PAGE en condición reductora con uso de DTT que evidenció una masa molecular de $23 \mathrm{kDa}$ y en condición no reductora una masa de $\sim 27 \mathrm{kDa}$. , para la determinación de la masa intacta del veneno recurrimos al método de Espectrometría de Masas obteniendo nuestros resultados crudos e intactos por Electrospray (ESI) dando un resultado de 27342.6641 Da. Se realizó una caracterización de la estructura primaria de "novo" por hidrolisis tríptica (MALDI/TOF) obteniendo 6 secuencias de aminoácidos para posteriormente realizar un análisis bioinformático a través de la base de datos SWISS-PROT para luego realizar un análisis de homología secuencial con uso del Software DNAStar especificando que la proteína estudiada es una metaloproteasa de la familia de las hemorraginas. Finalmente se hizo pruebas para su caracterización biológica para determinar la actividad hemorrágica con un método de inoculación intradérmica en ratones obteniendo los valores de hemorragia inducida de Bothrops roedingeri correspondiendo a $9.5 \mathrm{~mm} \pm 0.15 \mathrm{~mm}$ para el veneno total y de $8 \pm 0.12 \mathrm{~mm}$ para la fracción $\mathrm{HFBr}$, respectivamente.

\section{ABSTRACT}

The species belonging to the genus Bothrops are very important in the field of research for possessing proteolytic enzymes, which has as main characteristics to have a varied biological action and contribute to disorders in coagulation, necrosis and hemorrhage formation therefore a modern platform is needed in the study of these enzymes to be able to suggest an updated methodological model in the study of all the proteins expressed by a biological system that allows us to know the current status of the species, as well as to use this information in the biotechnological construction of information with possible future therapeutic purposes. The first objective is to purify and characterize one of the proteolytic enzymes present in the venom, such as metalloprotease, for which we initially worked with Hydrophobic Interaction chromatography with columns from the HITrap system, which resulted in the presence of 6 peaks, being peak 2 that showed hemorrhagic activity being called HFBr, with this obtained fraction SDS PAGE electrophoresis was performed in reductive condition using DTT that showed a molecular mass of $23 \mathrm{kDa}$ and in a non-reducing condition a mass of $\sim 27 \mathrm{kDa}$., for the determination of the intact mass of the poison we resorted to the method of Mass Spectrometry obtaining our raw and intact results by Electrospray (ESI) giving a result of $27342.6641 \mathrm{Da}$. A characterization of the primary structure of "novo" was carried out by tryptic hydrolysis (MALDI / TOF), obtaining 6 amino acid sequences to subsequently carry out a Bioinformatic analysis through the SWISSPROT database to then carry out a sequential homology analysis with Use of the DNAStar Software specifying that the protein studied is a Metalloprotease of the Bleeding family. Finally, tests were done for their biological characterization to determine the hemorrhagic activity with a method of intradermal inoculation in mice obtaining the values of induced hemorrhage of Bothrops roedingeri corresponding to $9.5 \mathrm{~mm} \pm 0.15 \mathrm{~mm}$ for the total venom and $8 \pm 0.12 \mathrm{~mm}$ for the fraction $\mathrm{HFBr}$, respectively. 


\section{INTRODUCCIÓN}

Los estudios de aproximación proteómica, son sin duda los primeros a ser realizados en nuestro país y como tal reviste de enorme trascendencia desde la generación de conocimiento hasta su posible aplicación. Se puede decir que hubo tres causas para el desarrollo de la proteómica:

- La secuenciación de genomas a gran escala y el desarrollo de bases de datos de proteínas.

- El desarrollo de técnicas de espectrometría de masas para analizar proteínas y péptidos.

- Los avances realizados en la separación de proteínas mediante electroforesis con la introducción de los gradientes de $\mathrm{pH}$ inmovilizados (IPGs).

La muestra a caracterizar es el veneno de Bothrops roedingeri, por la utilidad y características propiamente dichas que posee, una característica es que presenta proteínas con acción proteolítica (proteasas) dando a entender que su función principal es la descomposición de proteínas para aminoácidos, estas proteínas son llamadas metaloproteasas; una característica especifica es que necesita la presencia de un metal como el Zinc. Se tiene reportes que esta proteína está relacionada a los orígenes del cáncer debido a que su expresión está ubicada en altos niveles de tumores humanos y relacionados con la metástasis. Por eso, se menciona que puede tomar esta proteasa como tratamientos de cáncer para poder inhibir su expresión.

El veneno de serpiente perteneciente a la familia viperidae presenta alto porcentaje de metaloproteasas por ende tiene gran relevancia epidemiológica porque representan accidentes ofídicos de mayor incidencia en el Sur de América, pudiendo ocasionar frecuentemente disturbios funcionales permanentes e incluso la pérdida del miembro afectado.

El conocimiento de la presencia de metaloproteasas presentes en el veneno de serpientes, es común, sin embargo la presencia de esta familia de proteínas en el veneno de serpiente de Bothrops roedingeri, no es conocido, a pesar de algunos estudios previos, nos muestran su presencia pero para eso es necesario purificarla sin pérdida de la actividad biológica, con el fin de estudiarla de su perspectiva estructurafunción, y comprender no sólo algunos mecanismos de la patogénia, sino desde el punto de vista estructural pueda brindarnos información sobre el desarrollo futuro de un posible agente terapéutico.

El estudio realizado por espectrometría de masas está induciendo la reaparición de aproximaciones bioquímicas para el estudio de la función de las proteínas, como se mencionó para el análisis de un proteoma especifico se basará en electroforesis y cromatografías; para estos dos casos se continuará con espectrometría de masas como una cadena de metodologías debido a que existe una gran utilidad en la mejora y en el progreso de técnicas que nos mejorarán el estudio de proteínas y proteomas.

\section{METODOLOGÍA EXPERIMENTAL}

\section{Lugar de ejecución y recolección de muestras}

La muestra cruda de Bothrops Roendigeri fue cedida por el Prof. Luis Ponce Soto Ph.D. en forma cristalizada para poder empezar con la purificación en el laboratorio de Bioquímica de la Universidad Nacional de San Agustín (UNSA).

\section{Cromatografía de interacción hidrofóbica}

Las metaloproteasas, procedente del veneno de Bothrops roedingeri es purificada en columnas del sistema HITrap (H\&C USA.) El sistema cromatográfico usado es el de cromatografía convencional. Son recaudadas fracciones activas a una columna quelante HiTrap $(1 \mathrm{ml})$ que será cargada con 0,1 M de Bicarbonato de Amonio, se lavó con agua y se equilibró con Tampón de fosfato $0,05 \mathrm{M}, \mathrm{pH} 7,5$. Después las proteínas se eluyeron con un gradiente lineal (0$1.0 \mathrm{M}$ ) a un caudal de $60 \mathrm{ml} / \mathrm{h}$. El perfil de elución fue monitoreado a $280 \mathrm{~nm}$. Las fracciones que presentan mayor actividad metaloprotesasa y/o hemorrágica y son empleadas para la obtención del "pool" y guardadas a $0^{\circ} \mathrm{C}$.

\section{Actividad proteolítica de la metaloproteasa}

El substrato sintético, N-p-Tosyl-L-Argininametilester (TAME) fue utilizado para el dosaje de la muestra (Figura 6). Este sustrato ha sido empleado para medir la actividad amidásica para enzimas proteolíticas del tipo metaloproteasa. Estos substratos, péptidos derivados de p-Tosyl, han sido ampliamente utilizados principalmente por la alta sensibilidad fotométrica de la absorbancia a $405 \mathrm{~nm}$ de la p-Tosyl liberada después de la hidrólisis enzimática, según el método de ERLANGER.

El substrato cromogénico utilizado N-p-Tosyl-LArginina metil ester (TAME) $0,1 \mathrm{M}$ para el dosaje de la fracción $(1 \mathrm{mg} / \mathrm{ml}$.); $100 \mathrm{ul}$. de la muestra fueron colocados en un medio de incubación que contiene 1000 ul. de solución de sustrato previamente disuelto en Dymethyl sulfoxido como solución stock a ser utilizado en la proporción de 1/10 (10 ul.) en tampón Tris-HCl, (10 mm), CaCl2 (10 mm), NaCl, $(100 \mathrm{~mm}) \mathrm{pH}=7.8$, además de contener $250 \mathrm{ul}$. del mismo tampón. Para un volumen final de 1350 ul. Después de 30 minutos la reacción es bloqueada con ácido acético 30\% (500 U1.). Los cambios de absorbancia son leídos a $405 \mathrm{~nm}$.

\section{Electroforesis en SDS-PAGE}

SDS-PAGE se llevó a cabo de acuerdo con métodos descritos según Laemmli. Las muestras fueron pretratadas en condiciones reductoras (SDS más DTT) a $100^{\circ} \mathrm{C}$ durante 5 min. Los geles se tiñeron con $0.1 \%$ Coomassie azul brillante R-350 en etanol: ácido acético (5: 1, v / v) durante 15 minutos y se desvaneció en ácido acético al 10\%. La masa molecular se estimó por interpolación de un lineal gráfico logarítmico de masa molecular relativa versus distancia de la migración Marcadores de peso molecular estándar (Merck) fueron fosforilasa b $(97 \mathrm{kDa})$, albúmina bovina $(66 \mathrm{kDa})$, albúmina de huevo $(45 \mathrm{kDa})$, anhidrasa carbónica $(30 \mathrm{kDa})$ y a-lacta albumina de leche $(14 \mathrm{kDa})$. 


\section{Determinación de masa por espectrometría de masa Electrospray (ESI)}

La espectrometría de masas en Tandem ESI-CID-MS/MS fue elaborada empleando un espectrómetro de masas híbrido Quadrupolo-time of fligth (Q-TOF, Micromass Manchester, UK), suministrado con una fuente de nano spray operando en modo de ionización positivo. Las condiciones de ionización fueron: voltaje capilar de $2.3 \mathrm{kV}$, un cono voltaje y lentes RF1 de 30 y $100 \mathrm{~V}$, respectivamente y una energía de colisión de $10 \mathrm{eV}$. La temperatura de la fuente fue de $70^{\circ} \mathrm{C}$ y el cono de gas fue $\mathrm{N} 2$ a un flujo de $80 \mathrm{l} / \mathrm{h}$. El gas argonio para la fragmentación de los iones.

Fue realizada una calibración externa con yoduro sódico en una faja de masas de 50 a $3000 \mathrm{~m} / \mathrm{z}$. Todos los espectros fueron adquiridos con el analizador TOF en modo Vmode (TOF kV=9,1) y el voltaje MCP a $2150 \mathrm{~V}$.

Secuenciación "de novo" de péptidos trípticos por espectrometría de masa (MALDI TOF/TOF ABsciex 4800)

Los análisis de secuencias peptídicas para la caracterización de las proteínas serán realizados, mediante el programa PROTEIN PILOT.

La comparación cualitativa de múltiples muestras requiere algoritmos y métodos automáticos para el tratamiento de los datos, por lo que los programas MZmine 2 core y pkDACLASS, han sido seleccionados. Para los análisis de cuantificación libre de etiqueta se considerará el conteo espectral y la intensidad de picos mediante el uso de softwares libres.

\section{Estudio bioinformático de las enzimas}

Con la ayuda del software DNA Star, se procederá al estudio bioinformático de proteínas, el cual revelará informaciones de carácter estructural de las proteasas encontradas, utilizando específicamente EditSeq para importar y editar las secuencias con estos datos obtenidos y alineados con ayuda de MegAlign. Con esta información se ingresará a la base de datos de blast para observar la homología secuencial por medio de Blast (Swiss-prot: Annotated protein sequence database)

\section{Actividad hemorrágica}

\section{Método en piel}

Este procedimiento fue descrito originalmente por KONDO et al. ${ }^{[78]}$ empleando conejos. Sin embargo, el procedimiento ha sido modificado para ser empleado en ratas y ratones GUTIÉRREZ ${ }^{[79]}$. La técnica consiste en la inoculación intradérmica de veneno total, así como la fracción metaloproteasa esta se diluye en, agua desionizada en una concentración de $10 \mu \mathrm{g} / \mathrm{ml}$. en dos zonas del dorso previamente depilada del ratón. Al cabo de un determinado periodo de tiempo, los animales son sacrificados y se remueve la piel. Seguidamente, se mide el área hemorrágica del lado interno de la piel. La actividad hemorrágica se expresa como la "dosis hemorrágica mínima ", (DHM), para este caso es de $10 \mu \mathrm{g} / \mathrm{ml}$. e induce un área hemorrágica de $10 \mathrm{~mm}$ de diámetro. El análisis estadístico empleado fue de promedios incluyendo su desviación estándar.

\section{RESULTADOS \\ Cromatografía de interacción hidrofóbica}

El veneno total de Bothrops roedingeri de procedencia peruana, fue aplicado en una columna de interacción hidrofóbica y reveló la presencia de 6 picos, siendo el pico 2 aquel que mostró actividad hemorrágica y con una masa molecular de aproximadamente $23 \mathrm{kDa}$ en relación a los demás (Fig. 1).

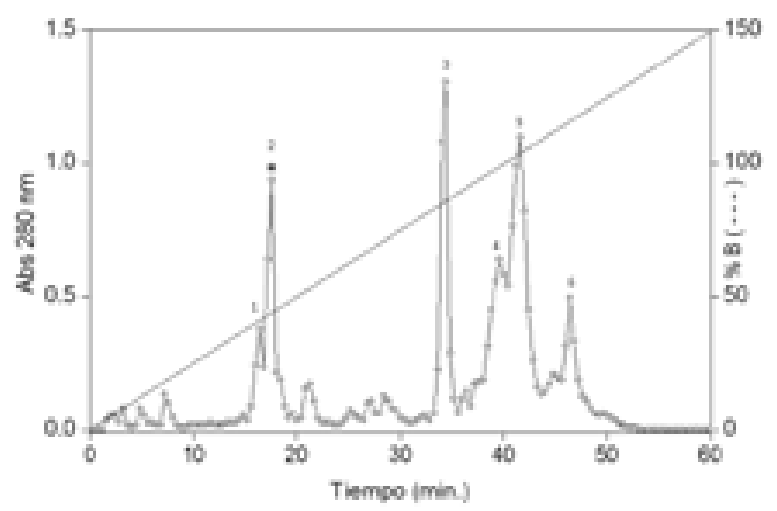

Fig.1: Cromatografía de interacción hidrofóbica, previamente equilibrada con 0,2mM de bicarbonato de amonio, $\mathrm{pH}$ 7.9. $10 \mathrm{mg}$. del veneno total de Bothrops roedingeri, fueron aplicados. Las fracciones fueron eluídas con un flujo de $10 \mathrm{ml} . / \mathrm{h}$. y con un gradiente linear $20 \mathrm{mM}$ a $1 \mathrm{M}$ de bicarbonato de amonio y las fracciones fueron colectadas y discriminadas por su actividad hemorrágica y con una masa molecular de aproximadamente $23 \mathrm{kDa}$, siendo registrado en el pico $2(*)$.

\section{Determinación de la actividad metaloproteasa}

La medida de actividad metaloproteasa para el veneno total de: Bothrops roedingerii es de 3,652 nmoles $/ \mathrm{min} . \pm$ 0,05 Para el caso de la fracción HFBr es: 7,51 nmoles/min. \pm 0,201 (Figura 2).

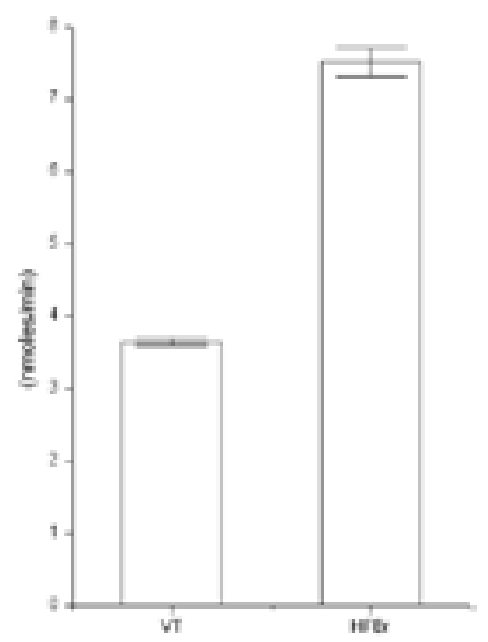

Fig.2: Medida de la actividad metaloproteasa de veneno total de Bothrops roedingerii y la fracción hemorrágica $\mathrm{HFBr}$ sobre el sustrato $\mathrm{Na}$-p-Tirosil-L-Arginina metil ester (TAME). 


\section{Electroforesis en SDS-PAGE}

Está mostrado en la Figura 3, donde la fracción indicada presenta una masa molecular de $23 \mathrm{kDa}$ aproximadamente, en condiciones reducidas, en la presencia de DTT y en condiciones evidencia una masa molecular de $\sim 27 \mathrm{kDa}$.

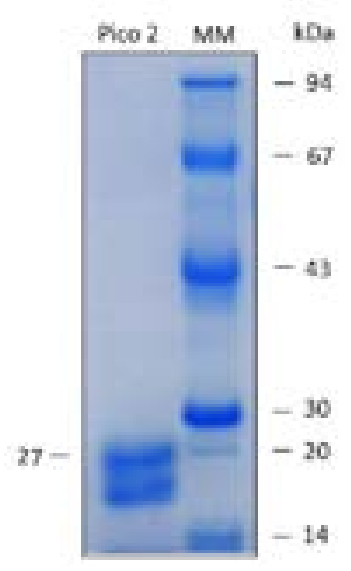

Fig. 3: Electroforesis en gel de poliacrilamida $12,5 \%(\mathrm{M} / \mathrm{V})$. $\mathrm{HFBr}$ aislada a partir de veneno total de Bothrops roedingerii; de procedencia peruana reducido. Mn: marcadores de masa molecular, coloreado con comassie blue.

Determinación de la masa intacta de HFBr de Bothrops roedingeri por espectrometría de masas MALDI TOF(ESI).

El análisis de la masa deconvolucionadas y cruda o intacta, por espectrometría de masas Electrospray (ESI), muestra ser de aproximadamente de 27342.6641 Da (Figura 4 y 5 respectivamente)

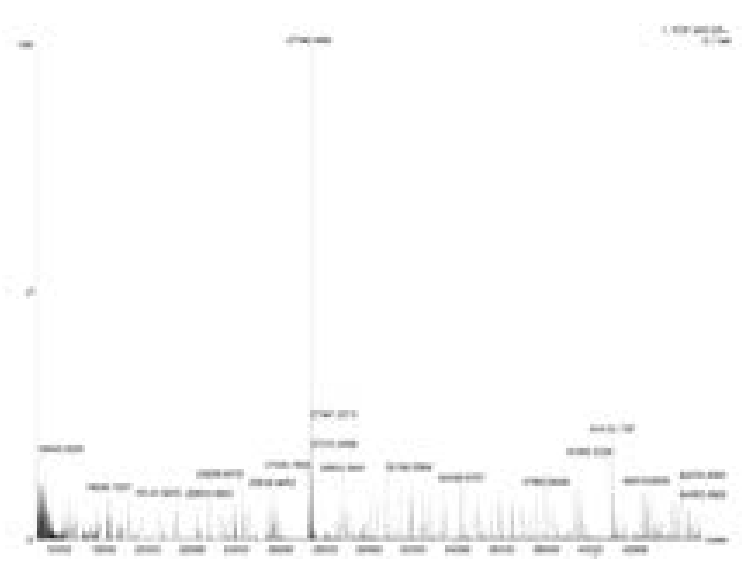

Fig. 4: Espectro de la masa deconvolucionada de la fracción $\mathrm{HFBr}$ procedente de Botrhops roedingeri por espectrometría de masas Electrospray (ESI

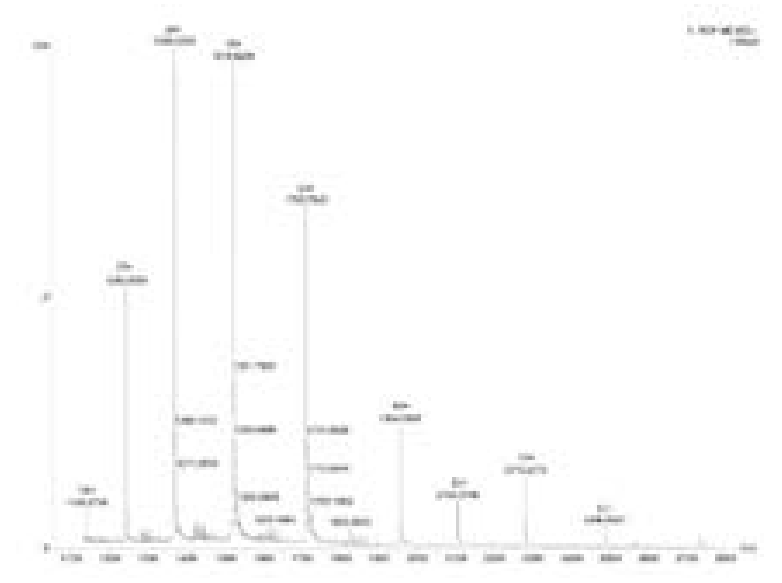

Fig. 5: Espectro de masa cruda e intacta de la fracción $\mathrm{HFBr}$ procedente de Botrhops roendingeri por espectrometría de masas Electrospray (ESI)

Caracterización de la estructura primaria de "novo" por espectrometría de masa MS/MS de la fracción $\mathrm{HFBr}$ procedente del veneno total de Bothrops roedingeri.

La proteína correspondiente al pico 2 del cromatograma e identificada como HFBr fue digerida con tripsina bovina y los péptidos trípticos resultantes fueron secuenciados por espectrometría de masa MS/MS. La tabla 1 muestra la masa observada de 7 péptidos trípticos, así como la secuencia del mismo y su posición en la secuencia deducida.

Tabla 1: Secuencia de aminoácidos de 7 péptidos obtenidos por hidrolisis tríptica a partir de la proteína correspondiente al pico 2 de la cromatografía de interacción hidrofóbica del veneno total de Bothrops roedingerii. La metionina fue modificada por oxidación.

\begin{tabular}{|c|c|c|c|}
\hline $\begin{array}{c}\text { Péptido } \\
\text { No. }\end{array}$ & $\begin{array}{c}\text { Masa } \\
\text { medida } \\
\text { (Da) }\end{array}$ & Secuencia de aminoácidos & $\begin{array}{c}\text { Masa } \\
\text { teórica } \\
\text { (Da) }\end{array}$ \\
\hline 1 & 673.82 & KFPQR & 673.78 \\
\hline 2 & 678.89 & SCIMAR & 678.85 \\
\hline 3 & 843.04 & DLIKVEK & 843 \\
\hline 5 & 1173.34 & LANLEVWSDK & 1173.3 \\
\hline 6 & 2238.48 & AHELGHNLGMDHDETCTCGAK & 2238.44 \\
\hline 7 & 3315.79 & SDHALEFSDCSVDYQTVGLAYTGPQCILRK & 3316.69 \\
\hline
\end{tabular}




\section{Estudio de homología secuencial(Análisis bioinformático), de la fracción 2 (HFBr) de Bothrops roedingeri.}

La figura 6 muestra los estudios de homología secuencial, por un análisis bioinformático, usando el software DNAStar (Ver 5.0), del factor hemorrágico HFBr procedente del veneno total de Bothrops roedingeri. La secuencia de los péptidos trípticos generados del pico 2 de la cromatografía de interacción hidrofóbica, a partir del veneno total de Bothrops roedingeri es sometido a un análisis de homología secuencial en la base de datos SWISS-PROT que revela que se trata de una proteína correspondiente a la familia de las hemorraginas procedentes del veneno de serpientes, disponibles en la base de datos consultada, con la que guarda una alto match o grado de homología secuencial con el péptido tríptico secuenciado por espectrometría de masa (MS/MS) (Figura 6)

Los trazos (- ), observados corresponden a los generados por el software para el proceso de alineamiento

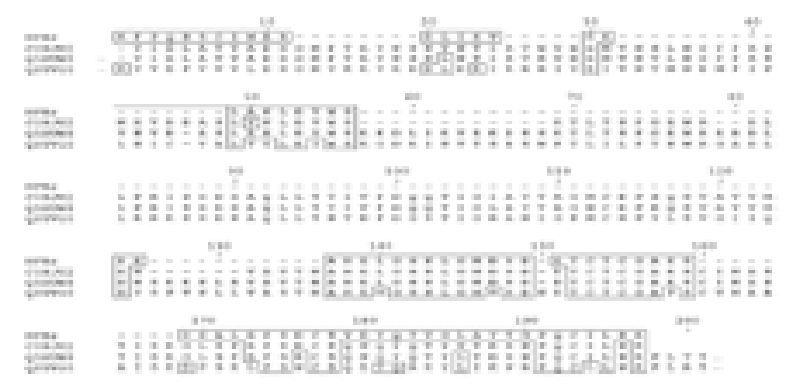

Fig. 6: Estudio comparativo de homología secuencial del factor hemorrágico $\mathrm{HFBr}$ procedente de Bothrops roedingeri con otras proteínas metaloproteasa provenientes de veneno de serpientes: BpMP-1 de Bothrops pauloensis (Accesion C0HJU2), Q5XUW8 de Bothrops insularis y Q8UVGO de Bothrops erythromelas

\section{Caracterización Biológica}

\section{Medida de la actividad hemorrágica}

La Figura 7 A y B muestra las dos zonas previamente depiladas, del ratón en las que son inoculadas intradérmicamente tanto veneno total, como la fracción $\mathrm{HFBr}$ proveniente de Bothrops roedingeri, además es inoculada con suero fisiológico ( $\mathrm{NaCl}$ 0,9\%) el que corresponde al control.

Fueron inoculadas 6 dosis de las cuales se seleccionó la dosis de $30 \mu \mathrm{g} / 0.1 \mathrm{ml}$ por ser las más representativa en el presente estudio

En la Figura 7 se muestra el sacrificio de los animales por punción cervical ya desangrados y preparados para ser removida la piel.

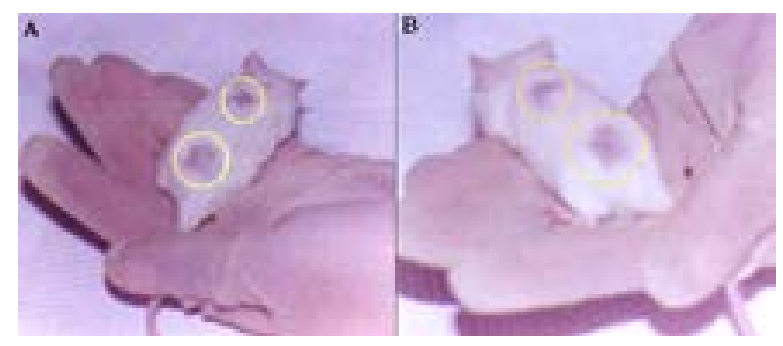

Fig. 7: Dorso depilado del ratón mostrando las dos zonas en las que se aplican las muestras respectivas intradermicamente en la dosis de $10 \square \mathrm{g} / \mathrm{ml}$.

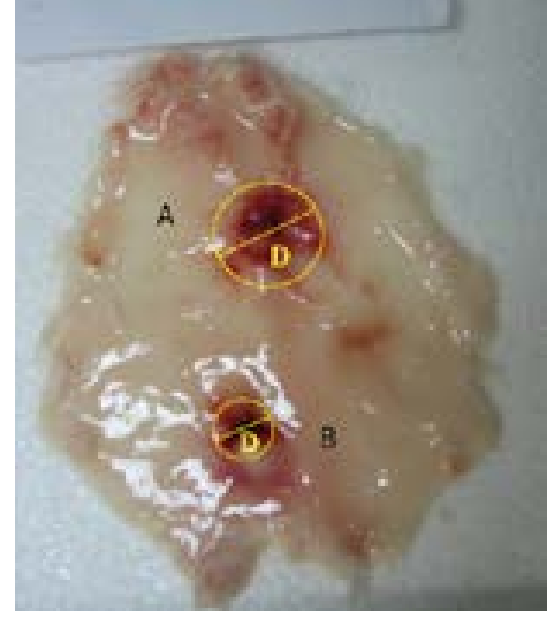

Fig. 8: Diametro de la hemorragia producida por veneno total de Bothrops roedingeri (B) y la fracción $\mathrm{HFBr}$ (A) luego de haber inoculado éste por vía parenteral

La actividad hemorrágica de los venenos de serpientes, así como de sus fracciones, se expresa como la "Dosis Hemorrágica Mínima" (DHM), definida como la dosis de veneno o fracción mínima que induce un área hemorrágica de $10 \mathrm{~mm}$ de diámetro.

Tabla 2: Valores de hemorragia inducida por el veneno total de Botrhops roedingerii y su fracción hemorrágica HFBr, en diferentes dosis.

\begin{tabular}{lcccccc}
\hline \multirow{6}{*}{ Dosis } & \multicolumn{5}{c}{ Medidas (mm) } \\
\cline { 2 - 6 } & \multicolumn{7}{c}{ HFBr } & \multicolumn{5}{c}{ Veneno Total } \\
\cline { 2 - 7 } & Diam1 & Diam2 & X1 & Diam1 & Diam2 & X2 \\
\hline $2,5 \mu \mathrm{g} / 0.1 \mathrm{ml}$ & 0 & 0 & 0 & 0 & 0 & 0 \\
$5 \mu \mathrm{g} / 0.1 \mathrm{ml}$ & 3 & 3 & 3 & 2 & 3 & 2.5 \\
$10 \mu \mathrm{g} / 0.1 \mathrm{ml}$ & 5 & 4 & 4.5 & 6 & 4 & 5 \\
$20 \mu \mathrm{g} / 0.1 \mathrm{ml}$ & 0 & 0 & 0 & 0 & 0 & 0 \\
$30 \mu \mathrm{g} / 0.1 \mathrm{ml}$ & 9 & 7 & 8 & 9 & 10 & 9.5 \\
$60 \mu \mathrm{g} / 0.1 \mathrm{ml}$ & 14 & 12 & 13 & 11 & 13 & 12 \\
\hline
\end{tabular}

\section{DISCUSIÓN}

La purificación de metaloproteasas a partir de veneno de serpientes ha sido largamente estudiado por la importancia de estas, desde el punto del envenenamiento ofídico, pues son capaces de producir una marcada actividad hemorrágica. Sin embargo, algunas enzimas fibrinolíticas altamente purificadas no producen actividad hemorrágica (MARKLAND, 1998).

Mahn, nos indica que la retención de proteínas en HIC es modelada a partir de la hidrofobicidad de proteínas y a la vez por las características del sistema, mediante modelos empíricos, pero el problema es que estos modelos son limitados porque no siempre se cuenta con información adecuada. 
Se ha conseguido purificar una metaloproteasa (HFBr) a partir de veneno total de Bothrops roedingeri. El fraccionamiento del veneno total se ha realizado en una técnica cromatográfica convencional de Interacción Hidrofóbica usando como fase estacionaria columnas quelantes (HITrap), dónde fue posible identificar la fracción correspondiente a la metaloproteasa la cual se ha identificado como el pico 2 , siendo considerada en base a los fundamentos como una proteína hidrofilica y de mayor actividad hemorrágica. La actividad enzimática ha sido posible monitorearla utilizando un sustrato cromogénico como es el $\mathrm{N} \square$-p-Tyrosil-LArginina metil ester (TAME) altamente específico para metaloproteinasas.

El proceso del fraccionamiento empleado nos ha ido revelando el grado de purificación de la metaloproteasa HFBr, pues la actividad se ha ido mostrando en aumento en la medida que hemos purificado, determinándose que la actividad metaloproteasa es dependiente de la pureza de la muestra. La técnica cromatográfica empleada nos ha revelado un alto grado de pureza, $u$ homogeneidad molecular como se ha podido establecer en la electroforesis en poliacrilamida (SDS-PAGE), además ha sido capaz de revelarnos su masa molecular relativa (Fig.4b) alrededor de $27 \mathrm{kDa}$.

Nuestra metaloprotesa HFBr ha mostrado ser una proteína con un fuerte carácter ácido conforme lo establecido en el análisis de aminoácidos, y su masa molecular 27342.6641 Da.

Desde la década de los 50 , cuando fue purificada la primera proteasa de veneno de serpiente, varios investigadores han ido reportando la purificación de esta clase de proteínas. Estas proteasas presentan actividad fibrinogenolítica, actuando en la coagulación sanguínea, fibrinólisis, agregación plaquetaria y en los procesos de trombosis y hemostasis (MATSUI, et al., 2000). Las metaloproteasas con actividad fibrinogenolítica son aplicables en los tratamientos médicos para bajar el nivel de fibrinógeno en el plasma o solubilizar los coágulos plasmáticos en individuos trombóticos (MATSUI, et al., 2000). Las proteasas de veneno de serpientes actúan en la conversión del fibronógeno el factor $\mathrm{V}, \mathrm{X}$; de la cascada de coagulación sanguínea, así como enzimas activadoras de la proteína. Estos son probablemente los inductores más importantes mediadores del daño y de los síntomas provocados por el veneno Bothropico.

En los venenos de serpientes encontramos una variedad de metaloproteasas que son altamente tóxicas, pudiendo o no causar hemorragia por interferir en la coagulación sanguínea y en la formación del tampón plaquetario, así como por degradar la membrana basal o los componentes de la matriz extracelular de sus víctimas.

A pesar de que han sido identificados muchos efectos biológicos y los responsables de tales efectos; la farmacología actual adopta ahora una nueva posición. Así un componente presente en el veneno que pudo ser capaz de causar un desequilibrio en la homeostasis, se convierte en un elemento valioso de utilidad biológica como puede ser el caso de algunas metaloproteinasas que al ser purificadas no produzcan hemorragia. Desde este punto de vista, los venenos de serpientes pasan a ser vistos desde otra perspectiva, como probables agentes terapéuticos en medicina, contra diversas patologías, así como herramientas o sondas moleculares en la investigación de diversos procesos fisiológicos.

La gran mayoría de metaloproteasas de veneno, hemorrágicas o no, son enzimas fibrinogenolíticas que clivan preferencialmente las cadenas alfa y posteriormente la beta del fibrinógeno (MARKLAND, 1998).

Debido al análisis Bioinformático (BLAST) se conoce que estas metaloproteasas purificadas pertenecen a la familia de las hemorraginas y por lo tanto tienen acción proteolíticas, se conoce que las hemorraginas se dividen en 4 subgrupos de acuerdo al peso molecular, las primeras metaloproteasas son las que tienen el peso molecular más bajo que se encuentra en un rango de 20 a $30 \mathrm{kDa}$ con poca o casi nula actividad hemorrágica, las de tipo II son toxinas de un tamaño mediano de 30 a $60 \mathrm{kDa}$ es muy similar a una desintegrina debido a sus dominios, el tipo III va más allá de una desintegrina y es rico en cisteína y el IV tipo es similar al tipo III solo que posee un dominio adicional de lectina.

El hecho de que nuestra metaloproteasa purificada $\mathrm{HFBr}$ a partir del veneno total de Bothrops roedingeri, posee actividad hemorrágica, nos lleva a consideración que esta podría estar siendo utilizada como un modelo biológico, como agente terapéutico antitrombótico, más existe una serie de caracterizaciones bioquímicas que precisarían ser evaluadas para poder confirmar su valiosa participación en estos procesos terapéuticos, tales como evaluar su actividad fibrinolítica y caracterizarla mejor a través de otras técnicas fisico-químicas como el secuenciamiento de su región $\mathrm{N}$-terminal, electroforesis bidimensional, afecto de agentes quelantes entre otras.

\section{AGRADECIMIENTOS}

Los autores agradecen al Vicerrectorado de Investigación de la Universidad Católica de Santa María que permitió desarrollar la investigación.

\section{REFERENCIAS BIBLIOGRÁFICAS}

1. Laemmli, U. K. (1970). Cleavage of structural proteins during the assembly of the head of bacteriophage T4. Nature 227(5259): 680-685.

2. Bjarnason, J. B.; Fox, J. W. Hemorrhagic metalloproteinase from snake venoms. Pharmac. Ther. V. 62, 1994.

3. Matsui T.; Fujimura Y.; Titani K. Snake venom proteases affecting hemostasis and thrombosis. Biochim. Biophys. Acta. v. 1477, 2000.

4. Kondo, H. et al. Studies on the quantitative method for the determination of hemorrhagic activity of Habu snake venom. Biol. v. 13, p. 43-51, 1960.

5. Gutiérrez, J.M. Neutralization of proteolytic and hemorrhagic activities of Costa Rican snake venoms by a polyvalent antivenom. Toxicon 23: 884-893, 1985

6. Erlanger B.F. and Goode L. Antibacterial activity of acyclic decapeptide analogs of gramicidin S. Science. 1961

7. Mahn A.V. Modelación Fenomenológica de Cromatografía de Interacción Hidrofóbica, Departamento de Ingeniería Química. Información Tecnológica Vol.20(3),135-144(2009)

8. Hjerten S., K. Yao, O. Eriksson y B. Johansson; Gradient and isocratic high - performance hydrophobic interaction chromatography of proteins on agarose columns. J. Chromatogr.: 359, 99- 109 (1986). 
9. Torres Huaco F., Ponce Soto LA, et al., Purification and Characterization of a New Weak Hemorrhagic Metalloproteinase BmHF-1 from Bothrops marajoensis Snake, Protein J (2010) 29,407-416

10. Graves P.R. Molecular biologist's guide to proteomics. Rev Mar; 66 2002. pp. 39-63

11. López-Lozano, J.L., de Sousa, M.V., Ricart, C.A., et al., Ontogenetic variation of metalloproteinases and plasma coagulant activity in venoms of wild Bothrops atroxspecimens from Amazonian rain forest. Toxicon 40, 2002.

12. Stroka A., Donato J.L. et.al. "Purification and characterization of a hemorrhagic metalloproteinase from Bothrops lanceolatus (Fer-de-lance) snake venom", Toxicon 45. pp 411-420 (2005)

13. Trummal, Katrin et al. MALDI-TOF mass spectrometry analysis of substrate specificity of lebetase, a directacting fibrinolitic metalloproteinase from Vipera lebetina snake venom. Biochim. Biophys. Acta. v. 1476, 2000.

14. Morandi V. Thrombospondin: Relationship to protease and growth factor/cytokine activity. Braz. J. Med. Biol. Res. v. 27, 1994.

15. Blundell T. L. Metalloproteinase super-families and drug design. Nature Struct. Biol. v. 1, pp 70-75, 1994. 